\title{
Caracterización de harinas de tritíceas híbridas
}

\author{
Castaño, M.N.; E.D. Ferrari, A.T. Picca, M.I. Curti, P.D. Ribotta, A.E. León, V.A. Ferreira, E.M. Grassi, \\ A. Ferreira, H.E. di Santo, E.A. Castillo y H.A. Paccapelo
}

\begin{abstract}
RESUMEN
Triticales ( $\mathrm{x}$ Triticosecale Wittmack) y tricepiros ( $\mathrm{x}$ Triticosecale Witt. $\mathrm{X} \times \mathrm{x}$ Agrotricum A. Camus) se mejoran con la finalidad de obtener cultivares de doble propósito (forraje y grano). Para su utilización en consumo humano, la calidad de las harinas de triticales es frecuentemente evaluada midiendo el diámetro y factor de expansión de las galletitas horneadas. Estos indicadores están fuertemente relacionados al contenido de almidón dañado, proteína y polisacáridos no amiláceos de la harina que determinan sus propiedades de absorción de agua. Se evaluaron 25 líneas experimentales, ocho cultivares de triticale y uno de tricepiro en Santa Rosa, La Pampa, Argentina, durante 2012, mediante análisis de correlación simple y de conglomerados. Se observaron correlaciones positivas entre los valores de la capacidad de retención del solvente agua, carbonato de sodio, ácido láctico y sacarosa, índice de retención de agua alcalina, pentosanos solubles y almidón dañado. La caracterización de las harinas y la separación en grupos según sus propiedades fisicoquímicas permitieron la selección de genotipos con diferentes propiedades funcionales. En el análisis de conglomerados, el cluster 1 agrupó los materiales con mejores características fisicoquímicas de las harinas, lo cual aumenta las posibilidades de su empleo en la alimentación humana.
\end{abstract}

Palabras clave: factor galletita, triticales, tricepiro, parámetros físico-químicos.

Castaño, M.N.; E.D. Ferrari, A.T. Picca, M.I. Curti, P.D. Ribotta, A.E. León, V.A. Ferreira, E.M. Grassi, A. Ferreira, H.E. di Santo, E.A. Castillo and H.A. Paccapelo 2017. Characterization of flour from hybrid triticeas. Agriscientia 34: 15-25

\section{SUMMARY}

Triticales ( $\mathrm{x}$ Triticosecale Wittmack) and Tricepiros ( $\mathrm{x}$ Triticosecale Witt. $X \times$ Agrotricum A. Camus) are improved in order to obtain dual-purpose cultivars (forage and grain). In order to use them for human consumption the quality of triticale flour is frequently evaluated by measuring the diameter and spread factor of cookies. These indicators are strongly related to the contents of damaged starch, protein and non-starch polysaccharides of flour, which determine its water absorption properties. During 2012, twenty-five experimental strains, eight triticale and one tricepiro cultivars were evaluated 
in Santa Rosa, La Pampa, Argentina by means of simple correlation and cluster analysis. Positive correlations between the values of solvent retention capacity of water, sodium carbonate, lactic acid and sucrose, retention rate of alkaline water, soluble pentosans and damaged starch were observed. The characterization of the tested flours and their differentiation based on their physicochemical properties allowed the selection of genotypes with different functional properties. In the cluster analysis, cluster 1 pooled the materials with the best physicochemical features of flour, which increases the possibilities of its use for human consumption.

Key words: cookie factor, triticales, tricepiro, physicochemical parameters.

M.N. Castaño, E.D. Ferrari, A.T. Picca y H. A. Paccapelo: Facultad de Agronomía, Universidad Nacional de La Pampa, RN $35 \mathrm{~km} 334$, (CP 6300), Santa Rosa, La Pampa, Argentina. M.I. Curti, P.D. Ribotta y A.E. León: Facultad de Ciencias Agropecuarias, UN Córdoba-CONICET, F.A.Marrone 746, (CP 5000), Córdoba, Argentina. V.A. Ferreira, E.M. Grassi, A. Ferreira, H.E. di Santo y E.A. Castillo: Facultad de Agronomía y Veterinaria, Universidad Nacional de Río Cuarto, RN 36 km 601 (CP 5800) Río Cuarto, Córdoba, Argentina. Correspondencia a: paccapelo@agro.unlpam.edu.ar

\section{INTRODUCCIÓN}

El triticale (x Triticosecale Wittmack) es un híbrido sintético proveniente de la cruza entre trigo (Triticum sp.) y centeno (Secale sp.). La rusticidad aportada por esta última especie permite su cultivo en regiones donde las condiciones climáticas adversas y la menor fertilidad de los suelos son restrictivas para el cultivo de trigo (Oettler, 2005). Tricepiro es el nombre vulgar aplicado por Covas (1976) a las combinaciones trigenéricas obtenidas a través de cruzamientos entre triticales ( $x$ Triticosecale Wittmack) y trigopiros (x Agrotricum A. Camus). En ellos se pretende optimizar la combinación entre trigo, centeno y agropiro, con la finalidad de reunir la calidad de uno, con la rusticidad de los otros. Este híbrido intergenérico puede constituirse en el segundo cereal sintético de características forrajeras en los sistemas de producción ganadera de la pampa subhúmeda, seca y semiárida. El uso principal del triticale en Argentina es como cereal forrajero; sin embargo, el grano de triticale puede convertirse en uno de suma importancia en la alimentación humana mediante el uso de su harina para la elaboración de pan integral y alimentos que no requieran harinas leudantes (Pérez et al., 2003; Rubiolo et al., 2004; Gómez et al., 2010; Oliete et al., 2010)

Para extender el uso del triticale a la alimentación humana se deben aumentar los rendimientos del cultivo y la calidad de las harinas. En Argentina la selección de genotipos de triticale con aptitud granífera para molienda se incorporó en forma relativamente reciente en programas de mejoramiento de las universidades nacionales de La Pampa y Río Cuarto, Argentina (Castro et al., 2011; Castaño et al., 2015 a, b; Ferreira et al., 2015). El mismo uso se podría esperar con las harinas de los tricepiros, de los cuales aún no se han publicado resultados por ser un cultivo muy incipiente.

Se han realizado análisis físicos y pruebas fisicoquímicas para predecir la calidad de las harinas, empleando parámetros tales como el índice de tamaño de partícula (PSI) (Método 55-30, AACC, 2000), el contenido de proteínas (Método 46-12, AACC, 2000), el índice de sedimentación en dodecil sulfato de sodio (IS-SDS) (método de Dick \& Quick, 1983), la prueba de Pelshenke (Método 5650, AACC 2000 ) y el índice de retención de agua alcalina (IRAA) (Método 56-10, AACC, 2000). Otros factores que afectan la calidad de las harinas de triticale para elaborar galletitas son el contenido de pentosanos (Roccia et al., 2006) y almidón dañado (Torri et al., 2003; Moiraghi et al., 2005; Roccia et al., 2006; Oliete et al., 2010).

Recientemente se ha desarrollado el perfil de capacidad de retención de solventes (SRC) (Método 56-11, AACC, 2000) para evaluar la calidad de los trigos blandos y es escasa la información de su aplicación en triticales (Ramírez et al., 2003; Roccia et al., 2006; Colombo et al., 2008). Esta prueba mide la habilidad de una harina para re- 
tener cuatro solventes diferentes (agua, sacarosa $50 \%$, carbonato de sodio 5\% y ácido láctico 5\%). Cada solvente predice la contribución funcional de los componentes de la harina: el SRC agua es afectado por todos los constituyentes hidrofílicos de la harina y se relaciona con la capacidad de retención de agua por los componentes poliméricos; el SRC láctico está asociado con la capacidad de formación de redes de la fracción de gluteninas y la fuerza del gluten; el SRC carbonato de sodio se vincula con el contenido de almidón dañado, y el SRC sacarosa está relacionado con el contenido de pentosanos accesibles a la fase líquida (Gaines, 2000; Guttieri et al., 2002; Kweon et al., 2009).

Las harinas para galletitas requieren baja capacidad de retención de agua (Fairidi et al., 1994), por ello se produce una mayor absorción de agua por parte del azúcar, se incrementa el contenido de jarabe y decrece la viscosidad de la masa durante el horneado. Esto permite una mayor extensión de la masa y la obtención de galletitas de mayor diámetro (Slade \& Levine, 1994). En Argentina se ha desarrollado un protocolo de horneado para la evaluación de galletitas dulces elaboradas con harina de triticale que permite determinar el factor galletita (León et al., 1996).

El objetivo de este trabajo fue caracterizar química y funcionalmente las harinas de líneas experimentales de tritíceas híbridas para identificar las de mejor calidad en la elaboración de galletitas.

\section{MATERIALES Y MÉTODOS}

\section{Materiales}

El presente trabajo se realizó en el Campo Experimental de la Facultad de Agronomía de la Universidad Nacional de La Pampa, Argentina, ubicado en Santa Rosa a $36^{\circ} 46^{\prime}$ S, 64 $4^{\circ} 17^{\prime}$ O, 210 m s.n.m., durante 2012. El suelo se clasificó como Paleoustol petrocálcico (Soil Survey Staff, 1999), con escasa pendiente superficial y un manto de tosca en el subsuelo, a una profundidad que varió entre 1,0 y 1,2 m. La siembra se efectuó el 9 de junio de 2012 y no se aplicó riego ni fertilizante. El total de precipitaciones durante el ciclo del cultivo fue de 524 $\mathrm{mm}$. La cosecha se efectuó manualmente a madurez comercial, permaneciendo el grano acondicionado hasta lograr aproximadamente 14\% de humedad.

En la Tabla 1 se detalla el nombre y procedencia de los materiales analizados. Comprende 22 líneas experimentales originadas en el Centro Internacional de Mejoramiento de Maíz y Trigo (CIMMYT) de México; tres de la Facultad de Agronomía y Vete- rinaria de la Universidad Nacional de Río Cuarto, Córdoba, Argentina; cuatro cultivares de triticales graníferos (Eronga 83, Cananea, GNU y Tatú); cuatro triticales forrajeros (Quiñé UNRC, Tizné UNRC, Don Santiago INTA y Yagán INTA) y un cultivar de tricepiro (Don René INTA).

\section{Análisis físico y fisicoquímico}

Para la obtención de las harinas los granos fueron molidos en un molino Quadrumat Junior Brabender (Método 26-50.01, AACC, 2000). Se determinó la dureza relativa de los granos mediante el índice de tamaño de partícula (PSI), (Método 5530, AACC, 2000). Los resultados fueron calculados como el peso relativo de harina tamizada $\times 100$ y luego comparados con una tabla para obtener la dureza relativa (Yamazaki \& Donelson, 1972).

En la predicción de la calidad y composición química de las harinas se utilizaron las siguientes técnicas:

1. Contenido total de proteínas, determinado por el método de Micro-Kjeldhal modificado para ácido bórico (Método 46-12, AACC, 2000).

2. Índice de sedimentación en dodecil sulfato de sodio (IS-SDS) (Método de Dick \& Quick, 1983).

3. Perfil de capacidad de retención de solventes (SRC) (Método 56-11, AACC, 2000).

4. Índice de retención de agua alcalina (IRAA) (Método 56-10, AACC, 2000).

5. Determinación del contenido de pentosanos. La cuantificación de pentosanos solubles se realizó siguiendo el método del Orcinol - $\mathrm{HCl}$ con las modificaciones realizadas por Hashimoto et al. (1987).

6. Almidón dañado (Método 76-30, AACC, 2000).

\section{Elaboración de galletitas}

Se elaboraron galletitas con las harinas en estudio. Los ingredientes utilizados fueron: $45 \mathrm{~g}$ de harina; $27 \mathrm{~g}$ de azúcar impalpable; 20,20 g de grasa vegetal; 2,25 g de leche en polvo; 0,50 g de bicarbonato de sodio; $0,42 \mathrm{~g}$ de sal y $8,5 \mathrm{ml}$ de agua. Las galletitas fueron horneadas a $180^{\circ} \mathrm{C}$ durante 10 minutos. La calidad se determinó mediante el factor galletita $(F G)$, obtenido de la relación entre el diámetro y la altura de cuatro galletitas orientadas al azar (León et al., 1996). 
Tabla 1. Nombre y procedencia de triticales y tricepiro cultivados en Santa Rosa, La Pampa, Argentina, durante 2012.

\begin{tabular}{|c|c|c|c|}
\hline & Nombre & Género & Procedencia \\
\hline 1 & LF53 x LF37 /12 & Triticale & Universidad Nacional de Río Cuarto (Córdoba, Argentina) \\
\hline 2 & $\begin{array}{l}\text { (T60 x Tehuelche) } \\
\text { x LF65 /6 }\end{array}$ & Triticale & Universidad Nacional de Río Cuarto (Córdoba, Argentina) \\
\hline 3 & LF97 x T312/11 & Triticale & Universidad Nacional de Río Cuarto (Córdoba, Argentina) \\
\hline 4 & Cim 03 FW/40 & Triticale & Centro Internacional de Mejoramiento de Maíz y Trigo (CIMMYT, México) \\
\hline 5 & Cim 03 FW/61 & Triticale & Centro Internacional de Mejoramiento de Maíz y Trigo (CIMMYT, México) \\
\hline 6 & Cim 03 FW/64 & Triticale & Centro Internacional de Mejoramiento de Maíz y Trigo (CIMMYT, México) \\
\hline 7 & Cim 03 FW/75 & Triticale & Centro Internacional de Mejoramiento de Maíz y Trigo (CIMMYT, México) \\
\hline 8 & Cim 03 FW/77 & Triticale & Centro Internacional de Mejoramiento de Maíz y Trigo (CIMMYT, México) \\
\hline 9 & Cim 03 IT/8 & Triticale & Centro Internacional de Mejoramiento de Maíz y Trigo (CIMMYT, México) \\
\hline 10 & Cim 03 IT/12 & Triticale & Centro Internacional de Mejoramiento de Maíz y Trigo (CIMMYT, México) \\
\hline 11 & Cim 05 IT/809 & Triticale & Centro Internacional de Mejoramiento de Maíz y Trigo (CIMMYT, México) \\
\hline 12 & Cim 05 IT/810 & Triticale & Centro Internacional de Mejoramiento de Maíz y Trigo (CIMMYT, México) \\
\hline 13 & Cim 05 IT/826 & Triticale & Centro Internacional de Mejoramiento de Maíz y Trigo (CIMMYT, México) \\
\hline 14 & Cim 05 IT/829 & Triticale & Centro Internacional de Mejoramiento de Maíz y Trigo (CIMMYT, México) \\
\hline 15 & Cim 05 IT/830 & Triticale & Centro Internacional de Mejoramiento de Maíz y Trigo (CIMMYT, México) \\
\hline 16 & Cim 05 IT/832 & Triticale & Centro Internacional de Mejoramiento de Maíz y Trigo (CIMMYT, México) \\
\hline 17 & Cim 05 IT/834 & Triticale & Centro Internacional de Mejoramiento de Maíz y Trigo (CIMMYT, México) \\
\hline 18 & Cim 05 IT/835 & Triticale & Centro Internacional de Mejoramiento de Maíz y Trigo (CIMMYT, México) \\
\hline 19 & Eronga 83 & Triticale & Centro Internacional de Mejoramiento de Maíz y Trigo (CIMMYT, México) \\
\hline 20 & Don Santiago-INTA & Triticale & Instituto Nacional de Tecnología Agropecuaria (INTA, Argentina ) \\
\hline 21 & Tizné-UNRC & Triticale & Universidad Nacional de Río Cuarto (Córdoba, Argentina) \\
\hline 22 & Quiñé-UNRC & Triticale & Universidad Nacional de Río Cuarto (Córdoba, Argentina) \\
\hline 23 & CIMMYT824 & Triticale & Centro Internacional de Mejoramiento de Maíz y Trigo (CIMMYT, México) \\
\hline 24 & CIMMYT 821 & Triticale & Centro Internacional de Mejoramiento de Maíz y Trigo (CIMMYT, México) \\
\hline 25 & CIMMYT 822 & Triticale & Centro Internacional de Mejoramiento de Maíz y Trigo (CIMMYT, México) \\
\hline 26 & CANANEA & Triticale & Centro Internacional de Mejoramiento de Maíz y Trigo (CIMMYT, México) \\
\hline 27 & CIMMYT 816 & Triticale & Centro Internacional de Mejoramiento de Maíz y Trigo (CIMMYT, México) \\
\hline 28 & CIMMYT 820 & Triticale & Centro Internacional de Mejoramiento de Maíz y Trigo (CIMMYT, México) \\
\hline 29 & Yagán INTA & Triticale & Instituto Nacional de Tecnología Agropecuaria (INTA, Argentina) \\
\hline 30 & Espiga Cuadrada & Triticale & Centro Internacional de Mejoramiento de Maíz y Trigo (CIMMYT, México) \\
\hline 31 & GNU & Triticale & Centro Internacional de Mejoramiento de Maíz y Trigo (CIMMYT, México) \\
\hline 32 & TATÚ & Triticale & Centro Internacional de Mejoramiento de Maíz y Trigo (CIMMYT, México) \\
\hline 33 & $\mathrm{C} 95 / 528$ & Triticale & Centro Internacional de Mejoramiento de Maíz y Trigo (CIMMYT, México) \\
\hline 34 & Don René INTA & Tricepiro & Instituto Nacional de Tecnología Agropecuaria (INTA, Argentina) \\
\hline
\end{tabular}

\section{Análisis estadístico}

Las determinaciones se realizaron por duplicado y se informaron como valor promedio \pm desvío estándar. Se analizaron las correlaciones entre parámetros a través de la prueba de Pearson ( ${ }^{*}$, ** nivel de significancia a $\mathrm{P}<0,05$ y $\mathrm{P}<0,01$, respectivamente) y se realizó un análisis de conglomerados de genotipos a partir de los valores promedios de las variables fisicoquímicas analizadas. El método jerárquico aglomerativo utilizado fue el de Ward con ligamiento promedio. Todos los análisis se realizaron con el software estadístico InfoStat (Di Rienzo et al., 2015).

\section{RESULTADOSY DISCUSION}

La dureza del grano es referida frecuentemente a la resistencia que opone al ser fracturado entre los rodillos del molino o a la energía requerida para reducir el endospermo a harina (Peña, 2003). Las texturas de los granos presentaron valores de PSI en un rango de 12,8 a 41,3 con un promedio de 26 (Tabla 2). Estos valores corresponden a las categorías de granos duros a extra blandos, respectivamente, según el método 55-30 de la American Association of Cereal Chemist (AACC, 2000) establecida para clasificar la textura de los diferentes granos de trigo. 
El grado de adhesión entre los componentes celulares del endospermo define qué tan duro es el endospermo de una variedad. Estas diferencias en la dureza del grano son de gran importancia ya que influyen de manera significativa en las propiedades de la molienda así como en la calidad de las harinas obtenidas (Miller et al., 1982).

La textura del grano de triticale se encuentra menos estudiada que la del grano de trigo. Muchas variedades de triticale presentan granos de textura blanda, similar a las de centeno, y sus harinas tienen un tamaño de partícula fina con valores de almidón dañado relativamente bajos (Ramírez et al., 2003).

Los resultados encontrados en este trabajo fueron levemente superiores a los publicados por Williams (1986) que informó un rango de dureza de 7,8 a 34,6 con coeficiente de variación de 35\% en 280 cultivares de triticale y a los de Rubiolo et al. (2004) con un rango de 7,4 a 27,4 en 25 líneas experimentales; a su vez, estos valores fueron considerablemente más bajos que los obtenidos para trigo por Olivera \& Baier (1991).

El contenido de proteína se situó en un rango de 6,9 a $12,1 \%$ con un promedio de $8,7 \%$; valores similares fueron publicados por Aguirre et al. (2002) (8,8 a 15,9\%) analizando 46 líneas experimentales de triticale en Córdoba. También Rubiolo et al. (2004) registraron un rango de entre 8,4 y 13,3\% analizando 25 líneas avanzadas. La calidad biológica de las proteínas está determinada por el contenido de aminoácidos esenciales, principalmente de lisina. El triticale posee un contenido de lisina promedio (3,7\% del total de proteínas) significativamente superior al de trigo (3\% del total de proteínas) (Guerrero García, 1999).

El índice de sedimentación en SDS mide las propiedades de hidratación y el grado de expansión de las proteínas, particularmente las gluteninas, las cuales se relacionan con la fuerza y extensibilidad del gluten. El volumen de sedimentación va a ser proporcional al contenido de proteínas formadoras de gluten y a su calidad (Vázquez, 2009). Los valores altos indican mejor calidad de harinas para panificación (Moiraghi et al., 2005). En este trabajo, los valores de SDS-SI variaron entre 5 y $10 \mathrm{~cm}^{3}$ con un promedio de $6,5 \mathrm{~cm}^{3}$. En la bibliografía se mencionan valores de entre 3,7 y $9,0 \mathrm{~cm}^{3}$ (Rubiolo et al., 2004); 5,0 y $11 \mathrm{~cm}^{3}$ (Ramírez et al., 2003) y 3,5 y $8,9 \mathrm{~cm} 3$ (Aguirre et al., 2002), todos considerablemente más bajos que los reportados para trigo por Olivera \& Baier (1991). Ello obedecería a que los triticales presentan una calidad panadera inferior, relacionada con una concentración más baja y una calidad más pobre de las proteínas del gluten (Amaya \& Peña, 1990; Rubiolo et al., 2004). Las proteínas de triticale contienen en promedio $28 \%$ de gliadinas y $32 \%$ de gluteninas; la concentración de gluteninas aumenta con el incremento de la concentración de proteínas en harinas, mientras que la concentración de gliadinas disminuye (Peña, 2003).

El índice de absorción de agua alcalina (IRAA) se aplica para predecir la calidad de una harina para elaborar galletitas. Las fracciones de pentosanos, proteínas, glicoproteínas y almidón dañado de las harinas son responsables de la retención de agua alcalina (Yamazaki \& Lord, 1971). Los valores obtenidos en este estudio variaron entre 72,3 y $87,8 \%$ con un promedio de $79,1 \%$ (Tabla 2 ). Ramírez et al. (2003) encontraron valores entre 59,5 y $68,1 \%$ con un promedio de $64,72 \%$ en líneas experimentales de triticales; Rubiolo et al. (2004) registraron valores de IRAA entre 59,7 y $72,5 \%$ mientras que Roccia et al. (2006) obtuvieron valores entre 62,5 y $74,2 \%$ y un promedio de $66,6 \%$. Una alta capacidad de retención de agua en las harinas resulta inconveniente para la extensibilidad de las galletitas, por lo tanto, harinas con valores de IRAA elevados son consideradas de mala calidad galletitera (León et al., 1996; Torri et al., 2003), mientras que bajos valores se traducen en galletitas de mayor diámetro (Gaines, 2000).

Los pentosanos son carbohidratos del tipo arabinoxilanos y, pese a ser componentes minoritarios en las harinas, han sido muy estudiados porque su presencia afecta la calidad de los productos panificados al incrementar la hidratación de las harinas (D’Appolonia \& Rayas-Duarte, 1994). En el presente trabajo, el contenido promedio de pentosanos solubles fue de $0,54 \%$ con una variación entre $0,05 \%$ y $1,29 \%$.

El perfil de capacidad de retención de solventes (SRC) es un método propuesto para evaluar la calidad de la harina y predecir el rendimiento panadero (AACC, 2000). Este perfil evalúa la habilidad de una harina para retener cuatro solventes diferentes (agua, carbonato de sodio 5\%, sacarosa $50 \%$ y ácido láctico 5\%) después de la centrifugación (Guttieri et al. 2002). Se encontró un amplio rango de valores dentro de cada perfil como se observa en la Tabla 3. Los promedios fueron de $75,9 \pm 8,7 \%$ (para agua); $103,7 \pm 11,7 \%$ para carbonatos; $112,7 \pm 7,8 \%$ para sacarosa y $96,4 \pm$ 13,9\% para ácido láctico. Los valores informados por Roccia et al. (2006) fueron inferiores a los del presente trabajo: $65,6 \%$ para agua; $80,8 \%$ (para carbonatos); $91,8 \%$ (para sacarosa) y $85,8 \%$ (para ácido láctico). Ramírez et al. (2003) mencionan valores promedios de 60,$1 ; 75,4 ; 96,8$ y $76,1 \%$ para los mismos solventes, respectivamente. Los valo- 
Tabla 2. Índice de tamaño de partícula (PSI), proteína, índice de sedimentación (SDS-SI), índice de retención de agua alcalina (IRAA) y pentosanos solubles (PS) de harinas de triticales y tricepiro cultivados en Santa Rosa, La Pampa, durante 2012.

\begin{tabular}{|c|c|c|c|c|c|c|}
\hline & Nombre & PSI & $\begin{array}{c}\text { Proteína } \\
(\%)\end{array}$ & $\begin{array}{c}\text { SDS - SI } \\
\left(\mathrm{cm}^{3}\right)\end{array}$ & $\begin{array}{c}\text { IRAA } \\
(\%)\end{array}$ & $\begin{array}{l}\text { PS } \\
(\%)\end{array}$ \\
\hline 1 & LF53 x LF37 /12 & $24,6 \pm 1,2$ & $8,7 \pm 0,5$ & $6,7 \pm 0,2$ & $74,4 \pm 0,3$ & $0,73 \pm 0,06$ \\
\hline 2 & (T60 x Teh.) $\times$ LF65 /6 & $14,1 \pm 0,9$ & $8,4 \pm 0,4$ & $6,5 \pm 0,1$ & $75,7 \pm 1,4$ & $0,68 \pm 0,01$ \\
\hline 3 & LF97 x T312/11 & $26,4 \pm 1,9$ & $7,8 \pm 0,3$ & $8,5 \pm 0,0$ & $80,0 \pm 0,1$ & $1,07 \pm 0,02$ \\
\hline 4 & Cim 03 FW/40 & $29,9 \pm 2,1$ & $7,6 \pm 0,3$ & $6,0 \pm 0,1$ & $87,8 \pm 1,8$ & $1,29 \pm 0,01$ \\
\hline 5 & Cim 03 FW/61 & $30,9 \pm 2,0$ & $7,3 \pm 0,6$ & $7,3 \pm 0,0$ & $82,6 \pm 0,4$ & $1,03 \pm 0,01$ \\
\hline 6 & Cim 03 FW/64 & $36,1 \pm 1,4$ & $8,0 \pm 0,2$ & $6,3 \pm 0,1$ & $81,7 \pm 1,0$ & $1,05 \pm 0,01$ \\
\hline 7 & Cim 03 FW/75 & $36,8 \pm 1,7$ & $7,6 \pm 0,2$ & $6,5 \pm 0,0$ & $75,9 \pm 0,3$ & $1,03 \pm 0,01$ \\
\hline 8 & Cim 03 FW/77 & $30,0 \pm 0,9$ & $7,0 \pm 0,0$ & $6,3 \pm 0,1$ & $79,6 \pm 0,0$ & $0,87 \pm 0,01$ \\
\hline 9 & Cim 03 IT/8 & $26,4 \pm 1,2$ & $9,2 \pm 0,4$ & $5,0 \pm 0,0$ & $76,0 \pm 0,9$ & $0,47 \pm 0,06$ \\
\hline 10 & Cim 03 IT/12 & $31,9 \pm 1,3$ & $9,0 \pm 0,4$ & $8,3 \pm 0,0$ & $80,7 \pm 0,4$ & $1,04 \pm 0,01$ \\
\hline 11 & Cim 05 IT/809 & $30,9 \pm 1,5$ & $9,1 \pm 0,0$ & $6,3 \pm 0,1$ & $83,1 \pm 1,6$ & $1,01 \pm 0,06$ \\
\hline 12 & Cim 05 IT/810 & $28,0 \pm 1,3$ & $7,4 \pm 0,1$ & $5,0 \pm 0,0$ & $76,1 \pm 0,3$ & $0,56 \pm 0,01$ \\
\hline 13 & Cim 05 IT/826 & $15,9 \pm 0,9$ & $7,9 \pm 0,0$ & $6,0 \pm 0,1$ & $79,2 \pm 1,7$ & $0,24 \pm 0,02$ \\
\hline 14 & Cim 05 IT/829 & $13,1 \pm 0,8$ & $7,4 \pm 0,1$ & $6,1 \pm 0,1$ & $80,4 \pm 0,5$ & $0,05 \pm 0,01$ \\
\hline 15 & Cim 05 IT/830 & $17,3 \pm 1,1$ & $9,0 \pm 0,7$ & $5,0 \pm 0,1$ & $72,3 \pm 0,9$ & $0,05 \pm 0,02$ \\
\hline 16 & Cim 05 IT/832 & $26,3 \pm 1,6$ & $6,9 \pm 1,1$ & $6,0 \pm 0,2$ & $74,90,4$ & $0,23 \pm 0,00$ \\
\hline 17 & Cim 05 IT/834 & $29,5 \pm 2,0$ & $7,6 \pm 1,0$ & $5,0 \pm 0,1$ & $77,8 \pm 2,6$ & $0,15 \pm 0,01$ \\
\hline 18 & Cim 05 IT/835 & $41,0 \pm 1,8$ & $7,0 \pm 5,3$ & $6,0 \pm 0,2$ & $80,6 \pm 0,1$ & $0,13 \pm 0,07$ \\
\hline 19 & Eronga 83 & $29,9 \pm 0,9$ & $1,4 \pm 0,1$ & $5,0 \pm 0,4$ & $77,0 \pm 0,1$ & $0,25 \pm 0,01$ \\
\hline 20 & Don Santiago-INTA & $26,5 \pm 1,1$ & $9,6 \pm 0,2$ & $6,5 \pm 0,5$ & $80,0 \pm 0,8$ & $0,42 \pm 0,05$ \\
\hline 21 & Tizné-UNRC & $31,0 \pm 1,3$ & $8,3 \pm 2,0$ & $7,3 \pm 0,4$ & $79,2 \pm 2,9$ & $0,74 \pm 0,03$ \\
\hline 22 & Quiñé-UNRC & $29,3 \pm 1,9$ & $8,2 \pm 2,0$ & $8,0 \pm 0,0$ & $81,0 \pm 0,4$ & $0,47 \pm 0,01$ \\
\hline 23 & CIMMYT824 & $15,0 \pm 1,8$ & $10,1 \pm 0,2$ & $10,0 \pm 0,0$ & $83,8 \pm 0,4$ & $0,24 \pm 0,08$ \\
\hline 24 & CIMMYT 821 & $20,3 \pm 0,9$ & $8,4 \pm 0,3$ & $6,0 \pm 0,7$ & $80,4 \pm 1,9$ & $0,81 \pm 0,10$ \\
\hline 25 & CIMMYT 822 & $21,7 \pm 1,1$ & $9,6 \pm 0,2$ & $7,0 \pm 0,2$ & $77,9 \pm 0,0$ & $0,27 \pm 0,18$ \\
\hline 26 & CANANEA & $25,3 \pm 1,5$ & $12,1 \pm 0,1$ & $6,0 \pm 0,7$ & $77,1 \pm 0,7$ & $0,52 \pm 0,04$ \\
\hline 27 & CIMMYT 816 & $22,6 \pm 1,6$ & $8,3 \pm 0,4$ & $6,8 \pm 0,1$ & $76,0 \pm 0,8$ & $0,28 \pm 0,02$ \\
\hline 28 & CIMMYT 820 & $27,0 \pm 1,4$ & $7,4 \pm 0,7$ & $6,0 \pm 0,2$ & $76,1 \pm 1,2$ & $0,38 \pm 0,08$ \\
\hline 29 & Yagán INTA & $41,3 \pm 1,9$ & $10,7 \pm 0,1$ & $10,0 \pm 0,1$ & $80,6 \pm 1,2$ & $0,35 \pm 0,10$ \\
\hline 30 & Espiga Cuadrada & $12,8 \pm 0,9$ & $10,3 \pm 0,1$ & $5,1 \pm 0,2$ & $78,4 \pm 1,0$ & $0,22 \pm 0,06$ \\
\hline 31 & GNU & $24,3 \pm 0.6$ & $9,6 \pm 0,1$ & $6,1 \pm 0,4$ & $77,8 \pm 0,3$ & $0,19 \pm 0,03$ \\
\hline 32 & TATÚ & $15,7 \pm 0,3$ & $9,6 \pm 0,0$ & $7,5 \pm 0,2$ & $80,3 \pm 1,8$ & $0,49 \pm 0,01$ \\
\hline 33 & C95/528 & $26,6 \pm 1,1$ & $8,8 \pm 0,2$ & $6,3 \pm 0,3$ & $87,4 \pm 3,9$ & $0,58 \pm 0,01$ \\
\hline \multirow[t]{3}{*}{34} & Don René INTA & $27,4 \pm 1,8$ & $11,0 \pm 0,4$ & $6,8 \pm 0,5$ & $77,8 \pm 0,7$ & $0,31 \pm 0,01$ \\
\hline & Promedio & 26,0 & 8,7 & 6,5 & 79,1 & 0,54 \\
\hline & Desvío Estándar & 7,41 & 1,3 & 1,2 & 3,4 & 0,36 \\
\hline
\end{tabular}

res de SRC dependen del nivel de extracción de la harina, puesto que un mayor nivel de extracción aumenta principalmente el nivel de SRC de carbonato de sodio y sacarosa (Guttieri \& Souza, 2003).

Cada solvente puede predecir la contribución funcional de los componentes de la harina: el SRCagua es afectado por todos los constituyentes hidrofílicos de la harina y se relaciona con la capacidad de retención de agua por los componentes poliméricos; el SRC-láctico está asociado con la capacidad de formación de redes de la fracción de gluteninas y la fuerza del gluten; el SRC-carbonato se vincula con el contenido de almidón dañado, y el SRC-sacarosa está relacionado con el contenido de pentosanos accesibles a la fase líquida (Gaines, 2000; Guttieri et al., 2002; Roccia et al., 2006; Kweon et al., 2009).

Los valores de almidón dañado variaron entre 7,4 y $14,4 \%$ con un promedio de $9,0 \%$. Ramírez et al. (2003) obtuvieron valores entre 6,92 y $9,44 \%$ analizando ocho genotipos de triticale y Oliete et al. (2010), valores entre 6,68 y $8,57 \%$. Se denomina almidón dañado a la fracción de gránulos de almidón rotos durante el proceso de obtención de 
las harinas (Torri et al., 2003) y puede ser utilizado para diferenciar entre trigos duros y blandos (Wade, 1988). La dureza del grano determina en buena medida la cantidad de almidón dañado presente en una harina y su posible destino (Hoseney, 1994). Los granos de textura blanda ofrecen una menor resistencia durante la molienda y la cantidad de almidón dañado disminuye (Giroux \& Morris, 1997) respecto a los granos de textura dura.

Como se mencionó anteriormente, la harina destinada a la elaboración de galletitas debe contener la menor cantidad posible de almidón dañado ya que reduce la capacidad de expansión de la galletita durante el horneado (Miller \& Hoseney, 1997). Por esta razón, la industria galletitera utiliza trigos blandos (suaves) que producen harinas con cantidades mínimas de almidón dañado.

Los valores obtenidos para el factor galletita oscilaron entre 4,5 (Tricepiro Don René INTA) y 7,3 (GNU). León et al. (1996) informaron factores

Tabla 3. Perfil de capacidad de retención de solventes (SRC), almidón dañado y factor galletita (FG) de harinas de triticales y tricepiros cultivados en Santa Rosa, La Pampa, durante 2012.

\begin{tabular}{|c|c|c|c|c|c|c|c|}
\hline \multirow{2}{*}{\multicolumn{2}{|c|}{ Nombre }} & \multicolumn{4}{|c|}{ Capacidad de Retención de Solventes (\%) } & \multirow{3}{*}{$\begin{array}{c}\begin{array}{c}\text { Almidón } \\
\text { Dañado (\%) }\end{array} \\
7,4 \pm 0,6\end{array}$} & \multirow{3}{*}{$\begin{array}{c}\begin{array}{c}\text { Factor } \\
\text { Galletita }\end{array} \\
5,8 \pm 0,2\end{array}$} \\
\hline & & \multirow{2}{*}{$\frac{\text { Agua }}{69,9 \pm 0,6}$} & \multirow{2}{*}{$\frac{\mathrm{Na}_{2} \mathrm{CO}_{3}}{95,4 \pm 0,8}$} & \multirow{2}{*}{$\begin{array}{r}\text { Sacarosa } \\
106,3 \pm 1,4\end{array}$} & \multirow{2}{*}{$\begin{array}{c}\text { Láctico } \\
92,2 \pm 1,1\end{array}$} & & \\
\hline 1 & LF53 x LF37 /12 & & & & & & \\
\hline 2 & $($ T60 x Teh. $\times$ LF65 /6 & $75,5 \pm 0,3$ & $109,3 \pm 0,9$ & $115,6 \pm 0,7$ & $104,8 \pm 1,2$ & $7,4 \pm 0,0$ & $6,0 \pm 0,1$ \\
\hline 3 & LF97 x T312/11 & $75,4 \pm 0,5$ & $97,6 \pm 0,5$ & $118,2 \pm 2.3$ & $127,1 \pm 0,9$ & $7,6 \pm 0,9$ & $5,8 \pm 0,1$ \\
\hline 4 & Cim 03 FW/40 & $95,6 \pm 0,3$ & $126,5 \pm 1,6$ & $125,8 \pm 1,4$ & $96,9 \pm 0,6$ & $9,5 \pm 0,6$ & $5,3 \pm 0,3$ \\
\hline 5 & Cim 03 FW/61 & $83,8 \pm 0,9$ & $119,8 \pm 2,1$ & $125,0 \pm 1,1$ & $102,5 \pm 0,8$ & $7,8 \pm 0,6$ & $5,6 \pm 0,9$ \\
\hline 6 & Cim 03 FW/64 & $78,1 \pm 0,8$ & $112,7 \pm 0,9$ & $113,3 \pm 1,7$ & $90,8 \pm 1,5$ & $8,1 \pm 0,3$ & $5,5 \pm 0,5$ \\
\hline 7 & Cim 03 FW/75 & $74,6 \pm 1,0$ & $95,0 \pm 1,4$ & $115,2 \pm 0,8$ & $111,0 \pm 1,4$ & $8,7 \pm 0,0$ & $5,2 \pm 0,2$ \\
\hline 8 & Cim 03 FW/77 & $74,7 \pm 0,4$ & $94,0 \pm 0,7$ & $108,7 \pm 0,1$ & $86,7 \pm 0,9$ & $8,3 \pm 0,6$ & $5,3 \pm 0,1$ \\
\hline 9 & Cim 03 IT/8 & $75,3 \pm 1,0$ & $96,2 \pm 1,5$ & $107,0 \pm 1,5$ & $80,5 \pm 0,5$ & $7,8 \pm 0,6$ & $5,5 \pm 0,3$ \\
\hline 10 & Cim 03 IT/12 & $77,2 \pm 0,9$ & $115,5 \pm 1,1$ & $113,5 \pm 0,4$ & $117,7 \pm 2,4$ & $8,3 \pm 0,6$ & $5,2 \pm 0,2$ \\
\hline 11 & Cim 05 IT/809 & $73,7 \pm 0,5$ & $97,9 \pm 0,9$ & $114,1 \pm 0,5$ & $99,6 \pm 2,1$ & $7,6 \pm 0,3$ & $6,0 \pm 0,2$ \\
\hline 12 & Cim 05 IT/810 & $74,5 \pm 1,3$ & $91,8 \pm 1,3$ & $107,5 \pm 1,2$ & $87,0 \pm 1,7$ & $8,0 \pm 0,9$ & $5,2 \pm 0,1$ \\
\hline 13 & Cim 05 IT/826 & $76,8 \pm 1,0$ & $97,3 \pm 1,3$ & $102,7 \pm 1,4$ & $92,4 \pm 1,6$ & $9,9 \pm 1,1$ & $5,5 \pm 0,1$ \\
\hline 14 & Cim 05 IT/829 & $79,3 \pm 0,8$ & $93,7 \pm 1,6$ & $114,2 \pm 0,6$ & $83,5 \pm 0,9$ & $9,5 \pm 0,5$ & $5,4 \pm 0,2$ \\
\hline 15 & Cim 05 IT/830 & $68,9 \pm 1,1$ & $94,8 \pm 1,1$ & $102,1 \pm 0,9$ & $76,5 \pm 1.3$ & $8,0 \pm 0,9$ & $6,3 \pm 0,2$ \\
\hline 16 & Cim 05 IT/832 & $72,8 \pm 1,4$ & $92,4 \pm 1,2$ & $104,4 \pm 0,4$ & $87,4 \pm 1,8$ & $8,9 \pm 0,3$ & $5,7 \pm 0,3$ \\
\hline 17 & Cim 05 IT/834 & $43,0 \pm 0,8$ & $91,4 \pm 0,8$ & $98,9 \pm 0,5$ & $85,6 \pm 2,0$ & $9,3 \pm 0,3$ & $6,1 \pm 0,1$ \\
\hline 18 & Cim 05 IT/835 & $73,7 \pm 1,6$ & $94,3 \pm 0,7$ & $103,6 \pm 1,2$ & $91,7 \pm 0,8$ & $8,9 \pm 0,8$ & $5,2 \pm 0,1$ \\
\hline 19 & Eronga 83 & $73,4 \pm 0,8$ & $93,3 \pm 1,0$ & $102,5 \pm 1,0$ & $87,5 \pm 1,2$ & $7,6 \pm 0,9$ & $5,6 \pm 0,3$ \\
\hline 20 & Don Santiago INTA & $96,6 \pm 1,4$ & $137,1 \pm 1,1$ & $124,1 \pm 0,9$ & $109,8 \pm 1,4$ & $14,4 \pm 0,6$ & $5,6 \pm 0,1$ \\
\hline 21 & Tizné-UNRC & $75,1 \pm 1,5$ & $123,1 \pm 0,9$ & $113,3 \pm 0,3$ & $106,9 \pm 1,7$ & $10,3 \pm 0,5$ & $5,4 \pm 0,1$ \\
\hline 22 & Quiñé-UNRC & $81,8 \pm 1,5$ & $108,0 \pm 0,6$ & $122,3 \pm 1,4$ & $105,6 \pm 0,9$ & $7,6 \pm 1,5$ & $6,0 \pm 0,2$ \\
\hline 23 & CIMMYT824 & $74,0 \pm 0,9$ & $101,8 \pm 1,2$ & $108,1 \pm 2,1$ & $87,4 \pm 1,2$ & $8,2 \pm 1,7$ & $5,6 \pm 0,2$ \\
\hline 24 & CIMMYT 821 & $74,5 \pm 0,7$ & $96,2 \pm 1,7$ & $112,7 \pm 2,3$ & $85,7 \pm 1,8$ & $9,9 \pm 0,0$ & $5,5 \pm 0,1$ \\
\hline 25 & CIMMYT 822 & $69,6 \pm 1,0$ & $93,5 \pm 1,6$ & $108,1 \pm 1,4$ & $84,1 \pm 0,9$ & $9,3 \pm 0,8$ & $5,4 \pm 0,3$ \\
\hline 26 & CANANEA & $75,8 \pm 0,8$ & $96,9 \pm 2,1$ & $113,7 \pm 1,2$ & $91,6 \pm 1,1$ & $9,3 \pm 0,3$ & $6,5 \pm 0,4$ \\
\hline 27 & CIMMYT 816 & $72,1 \pm 1,0$ & $99,9 \pm 1,7$ & $114,1 \pm 1,1$ & $99,2 \pm 0,8$ & $8,7 \pm 0,6$ & $6,0 \pm 0,1$ \\
\hline 28 & CIMMYT 820 & $72,2 \pm 0,9$ & $96,8 \pm 1,5$ & $108,3 \pm 1,4$ & $90,0 \pm 2,3$ & $10,1 \pm 0,3$ & $6,4 \pm 0,2$ \\
\hline 29 & Yagán INTA & $78,4 \pm 2,1$ & $116,4 \pm 2,3$ & $133,4 \pm 0,9$ & $133,0 \pm 2,5$ & $7,5 \pm 0,0$ & $5,3 \pm 0,2$ \\
\hline 30 & Espiga Cuadrada & $74,6 \pm 0,8$ & $104,6 \pm 1,9$ & $115,0 \pm 1,0$ & $74,8 \pm 1,7$ & $11,1 \pm 0,0$ & $5,4 \pm 0,1$ \\
\hline 31 & GNU & $73,7 \pm 1,8$ & $107,1 \pm 1,5$ & $108,1 \pm 1,2$ & $81,1 \pm 1,4$ & $9,5 \pm 0,6$ & $7,3 \pm 0,2$ \\
\hline 32 & TATÚ & $77,0 \pm 1,1$ & $102,1 \pm 0,9$ & $114,4 \pm 2,2$ & $112,4 \pm 1,2$ & $9,9 \pm 0,0$ & $5,5 \pm 0,1$ \\
\hline 33 & C95/528 & $93,0 \pm 0,8$ & $122,4 \pm 1,0$ & $123,9 \pm 1,6$ & $108,2 \pm 1,6$ & $13,2 \pm 0,6$ & $5,4 \pm 0,1$ \\
\hline \multirow[t]{3}{*}{34} & Don Rene INTA & $75,0 \pm 2,1$ & $109,3 \pm 2,1$ & $113,7 \pm 1,0$ & $106,4 \pm 2,1$ & $9,1 \pm 1,7$ & $4,5 \pm 0,3$ \\
\hline & Promedio & 75,9 & 103,7 & 112,7 & 96,4 & 9,0 & 5,6 \\
\hline & Desvío Estándar & 8,7 & 11,7 & 7,8 & 13,9 & 1,4 & 0,49 \\
\hline
\end{tabular}


galletitas de 6 para Cananea; 5,7 para Eronga 83; 5,4 para Yagán INTA y 5,2 para Quiñé-UNRC, siendo el primero de aceptable calidad, el segundo y el tercero de dudosa calidad y el último de mala calidad, de acuerdo a las categorías usadas por el CIMMYT. En el presente trabajo, los genotipos 32 (GNU), 26 (CANANEA), 28 (Cimmyt 820), 15 (Cim 05 IT/830), 17 (Cim 05 IT/834), 2 (T60 x Teh.) x LF65/6 y 22 (Quiñé-UNRC) presentaron valores de FG igual o superiores a 6 .

\section{Relación entre parámetros de la calidad de harinas}

La Tabla 4 resume las correlaciones entre los parámetros de calidad de las harinas. Los valores de índice de tamaño de partícula asociaron positivamente con el porcentaje de SRC ácido láctico y pentosanos solubles, indicando que a medida que se incrementa la textura blanda del grano se incrementan los carbohidratos correspondientes a la fracción de pentosanos solubles.

Tabla 4. Correlaciones entre parámetros fisicoquímicos obtenidos de harinas de triticale y tricepiro cultivados en Santa Rosa, La Pampa, durante 2012.

\begin{tabular}{|c|c|c|c|c|c|c|c|c|c|c|}
\hline & & & & & Capacida & d de Retenci & ión de Solvente & $(\%)$ & & \\
\hline & PSI & Prot. (\%) & SDS - SI & IRAA & Agua (\%) & $\mathrm{Na}_{2} \mathrm{CO}_{3}(\%)$ & Sacarosa (\%) & Láctico (\%) & P. S. & A.D. \\
\hline$\overline{\mathrm{PSI}}$ & 1,00 & & & & & & & & & \\
\hline Prot. (\%) & $-0,15$ & 1,00 & & & & & & & & \\
\hline SDS - SI & 0,14 & 0,16 & 1,00 & & & & & & & \\
\hline IRAA & 0,18 & $-0,06$ & $0,37^{*}$ & 1,00 & & & & & & \\
\hline Agua (\%) & 0,06 & 0,05 & 0,20 & 0,56 ** & 1,00 & & & & & \\
\hline $\mathrm{Na}_{2} \mathrm{CO}_{3}(\%)$ & 0,20 & 0,13 & $0,33^{*}$ & $0,55^{\star *}$ & $0,70^{\star *}$ & 1,00 & & & & \\
\hline Sacarosa (\%) & 0,23 & 0,12 & $0,51^{* *}$ & $0,57^{\star \star}$ & $0,71^{* *}$ & $0,73^{\text {** }}$ & 1,00 & & & \\
\hline Láctico (\%) & $0,40^{* *}$ & 0,07 & $0,68^{* *}$ & 0,31 & $0,35^{*}$ & 0,50 ** & $0,68^{* *}$ & 1,00 & & \\
\hline P. S. & $0,37^{\star}$ & $-0,26$ & 0,20 & $0,41^{* *}$ & $0,36^{*}$ & $0,35^{*}$ & $0,44^{* *}$ & $0,43^{* *}$ & 1,00 & \\
\hline A.D. & $-0,20$ & 0,08 & $-0,19$ & 0,28 & $0,41^{* *}$ & $0,47^{* *}$ & 0,20 & $-0,01$ & $-0,19$ & 1,00 \\
\hline$F G$ & $-0,22$ & 0,07 & $-0,14$ & $-0,28$ & $-0,30$ & $-0,22$ & $-0,23$ & $-0,29$ & $-0,20$ & $-0,14$ \\
\hline
\end{tabular}

El índice de sedimentación en dodecil sulfato de sodio estuvo asociado positivamente al índice de retención de agua alcalina y a la capacidad de retención de los solventes carbonatos, sacarosa y láctico. Esto sugiere que a medida que aumenta la proporción de gluteninas de alto peso molecular aumentan los carbohidratos del tipo de pentosanos solubles; también se produce un incremento del almidón dañado y, como consecuencia, se incrementa el contenido de agua alcalina.

El índice de retención de agua alcalina se encontró asociado con la capacidad de retención de los solventes agua, carbonatos y sacarosa y con los pentosanos solubles. Estos resultados indicarían que parte del agua alcalina retenida por una harina puede ser explicada por la presencia de almidón dañado y la influencia de los pentosanos (Yamazaki \& Lord, 1971). En investigaciones previas se mencionan correlaciones más altas entre dichos parámetros (Rubiolo et al., 2004; Roccia et al., 2006; Colombo et al., 2008, Duyvejonck et al., 2012).

Se encontraron asociaciones positivas y significativas entre la capacidad de retención de los distintos solventes (agua, carbonato, sacarosa y láctico). Todos correlacionaron positivamente con las fracciones de pentosanos solubles y los dos primeros con almidón dañado. Un aumento del almidón dañado y de pentosanos incrementa la absorción total de agua (Roccia et al., 2006). El almidón dañado afecta la hidratación de las harinas llegando a absorber hasta tres veces más que el almidón sano (Bushuk, 1998).

La naturaleza altamente hidrofílica de los pentosanos solubles puede incrementar hasta diez veces su peso en agua (Bloksma \& Bushuk, 1988). Las harinas destinadas a la elaboración de galletitas deben absorber la menor cantidad de agua posible, ya que la masa pegajosa no permite el paso a través de las máquinas de laminado y recorte; además, deben ser horneadas durante más tiempo para evaporar el exceso de humedad, aumentando el costo de energía (Pantanelli, 2002).

La correlación positiva entre la capacidad de retención de ácido láctico y la capacidad de retención de sacarosa también fue encontrada para trigos blandos en otros trabajos (Guttieri et al., 2002; Guttieri \& Souza, 2003), en donde se sugirió que la asociación alcanzada podría ser debida a características propias de dos de las líneas en estudio, más que a una propiedad universal de los trigos o de la capacidad de retención de los solventes. 
La correlación observada entre la capacidad de retención de sacarosa y carbonato de sodio coincide con lo informado en trabajos anteriores para trigos blandos (Guttieri \& Souza, 2003) y triticale (Roccia et al., 2006). No se encontró asociación entre la capacidad de retención de ácido láctico y el contenido de proteínas, coincidente con lo observado en 26 cultivares de trigo por Guttieri et al. (2002) y Rubiolo et al. (2004) en 25 líneas avanzadas de triticale.

\section{Análisis de conglomerados}

Se evaluó, mediante un análisis de conglomerados, la influencia de la composición química y las propiedades fisicoquímicas de las muestras de harinas y su relación con la calidad galletitera. El análisis estadístico permitió diferenciar las harinas en tres grupos; grupo $1(17,27,25,14,28,11,31$, $32,24,6,18,19,16,13,15,9,12,8,26$ y 1), grupo $2(29,23,22,5,33,7,3,21,10,30,2)$ y grupo 3 $(20,34,4)$ (Tabla 5). Estos mostraron diferencias significativas en sus propiedades de absorción de distintos solventes. En general, altos valores de absorción se relacionaron con mayores contenidos de almidón dañado y pentosanos solubles, y no mostraron relación con el contenido de proteínas de las harinas (Fig. 1).

Las muestras incluidas en el grupo 1 mostraron el mayor factor galletita asociado con bajos valores de los porcentajes de la capacidad de retención

Tabla 5. Clasificación de harinas de triticales y tricepiros cultivados en Santa Rosa, La Pampa, durante 2012 según análisis de conglomerados

\begin{tabular}{|c|c|c|c|c|c|c|c|c|}
\hline & \multirow{2}{*}{ IRAA } & \multicolumn{4}{|c|}{ Capacidad de retención de solventes } & \multirow{2}{*}{$\begin{array}{c}\text { Almidón } \\
\text { Dañado \% }\end{array}$} & \multirow{2}{*}{$\begin{array}{c}\text { Factor } \\
\text { Galletita }\end{array}$} & \multirow{2}{*}{ SDS - SI } \\
\hline & & Agua (\%) & $\mathrm{Na2C03( \% )}$ & Sacarosa (\%) & Ácido Láctico (\%) & & & \\
\hline 1 * & $78,1 \mathrm{a}$ & $72,4 \mathrm{a}$ & $97,2 \mathrm{a}$ & $108,3 \mathrm{a}$ & $87,4 \mathrm{a}$ & 8,9 a & $5,8 \mathrm{~b}$ & $6,4 \mathrm{a}$ \\
\hline Conglomerado $2^{* *}$ & $79,5 \mathrm{a}$ & $77,6 \mathrm{~b}$ & $109,5 b$ & $118,6 b$ & $112,4 \mathrm{c}$ & $8,4 \mathrm{a}$ & $5,5 \mathrm{a}$ & $8,0 \mathrm{~b}$ \\
\hline Conglomerado 3 *** & $85,1 \mathrm{~b}$ & $95,1 \mathrm{c}$ & $128,7 \mathrm{c}$ & $124,6 \mathrm{c}$ & $105,0 \mathrm{~b}$ & $12,4 \mathrm{~b}$ & $5,4 \mathrm{a}$ & $6,4 \mathrm{a}$ \\
\hline
\end{tabular}

Los datos se expresan como el valor promedio para cada conglomerado. Letras distintas en una misma columna indican diferencias significativas $(p \leq 0,05)$. IRAA: Índice de retención de agua alcalina; SDS-SI: Índice de sedimentación en dodecil sulfato de sodio.

* Líneas: $1,26,8,12,9,15,13,16,19,18,6,24,32,31,11,28,14,25,27,17$

** Líneas: $2,30,10,21,3,7,33,5,22,23,29$

*** Líneas: 4, 34, 20

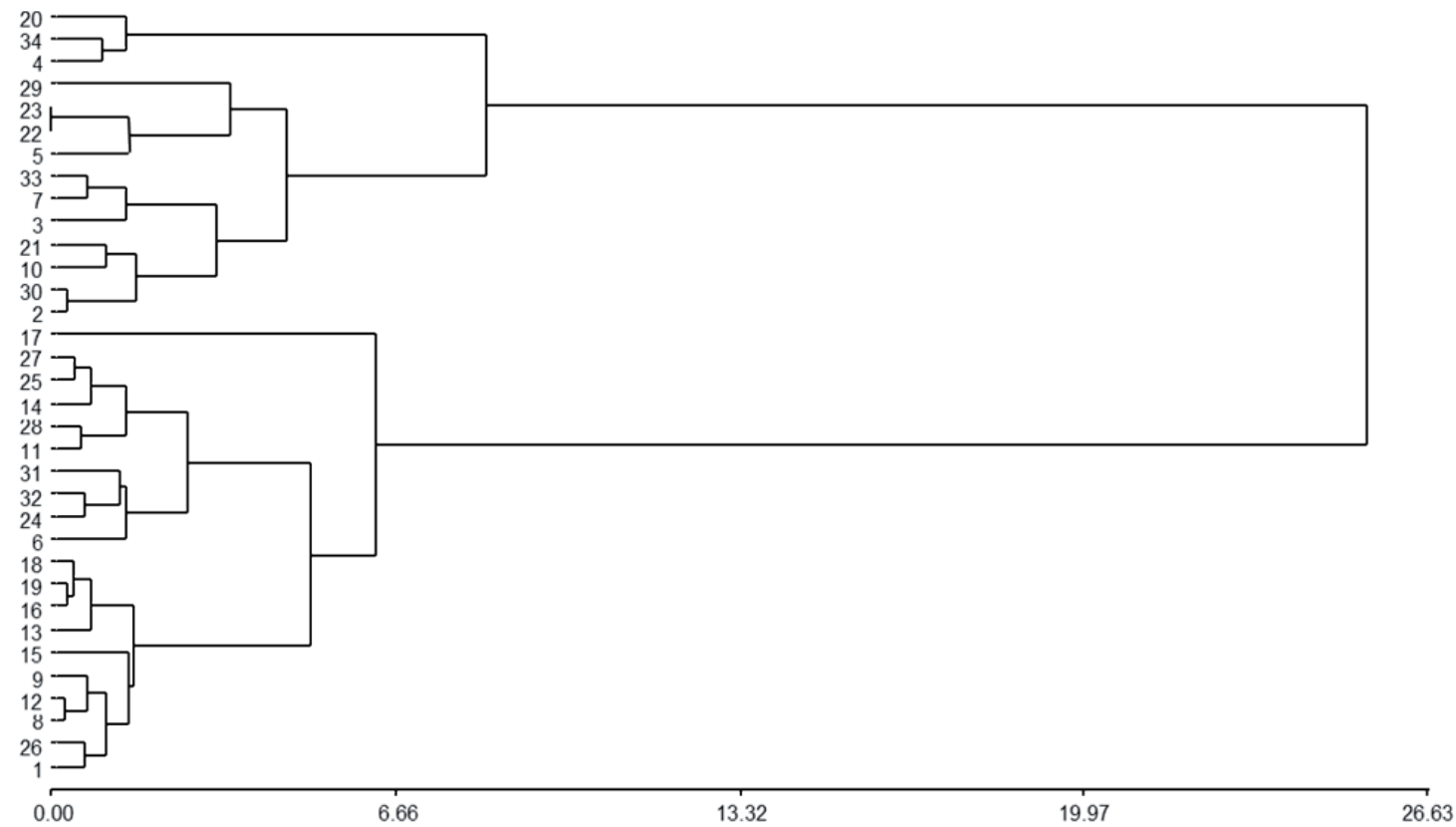

Figura 1. Análisis de conglomerados de genotipos de triticale y tricepiro cultivados en Santa Rosa, La Pampa, durante 2012, sobre la base de los valores promedios de las variables fisicoquímicas obtenidas de sus harinas. 
de solventes, índice de retención de agua alcalina, índice de sedimentación en dodecil sulfato de sodio, pentosanos solubles y almidón dañado. El grupo 3 fue el que incluyó la menor cantidad de muestras siendo estas las que obtuvieron mayores valores en los indicadores de absorción, lo que es consecuente con el menor factor galletita. En este grupo se encontró la muestra 34 correspondiente a la variedad de tricepiro analizada, lo que indicaría que no presenta aptitud para su utilización en la elaboración de galletitas.

\section{CONCLUSIONES}

La caracterización de las harinas y la separación en grupos según sus propiedades fisicoquímicas permitieron la selección de genotipos con diferentes propiedades funcionales. Las muestras incluidas en el cluster 1 presentaron los mejores parámetros de calidad relacionados con la elaboración de galletitas. El tricepiro presentó el menor valor de factor galletita.

\section{BIBLIOGRAFÍA}

Aguirre, A.; O. Badiali, M. Cantarero, A. León, P. Ribotta y O. Rubiolo, 2002. Relationship of test weight and kernel properties to milling and baking quality in argentine triticales. Cereal Res. Comm. 30:203-208.

Amaya, A. y R. Peña, 1990. Triticale industrial quality improvement at CIMMYT. Past, present and future. In: Proc. $2^{\text {nd }}$ Int. Triticale Symp. pp 412-421. Berthier Grafica Editora. Passo Fundo, Brasil.

American Association of Cereal Chemist (AACC), 2000. Approved Methods of the AACC. 10 $0^{\text {th }}$ Edition, American Assoc. Cereal Chemists Inc. , St Paul, Minnesota. USA.

Bloksma, A. and W. Bushuk, 1988. Rheology and chemistry of dough. En Y. Pomeranz (Ed.): Wheat: chemistry and technology. pp 131-217. American Assoc. Cereal Chemists Inc., St Paul, Minnesota. USA.

Bushuk, W. 1998, Interactions in wheat doughs. In Harmer, R. \& R. Hoseney (Eds): Interactions: The keys to cereal quality. pp 1-16. American Association of Cereal Chemists Inc., St. Paul, Minnesota, USA.

Castaño, M.; P. Ribotta, V. Ferreira, E. Grassi, A. Ferreira, H. di Santo, E. Castillo, y H. Paccapelo, 2015a. Aptitud de las harinas integrales de triticales (x Triticosecale Wittmack) para la elaboración de galletitas. SEMIÁRIDA Rev. Facultad de Agronomía, UN La Pampa 25(1):25-39.

Castaño, M.; P. Ribotta, V. Ferreira, E. Grassi, A. Ferreira, H. di Santo, E. Castillo y H. Paccapelo, 2015b. Análisis del perfil fisicoquímico de las harinas de triticales ( $x$ Triticosecale Wittmack) y su relación con la elaboración de galletitas de calidad. Rev. SENASA 1(9):1-14.

Castro, N.; R. Domínguez, y H. Paccapelo, 2011. Análisis del rendimiento de grano y sus componentes en cereales sintéticos (tricepiros y triticales). Rev. Facultad de Agronomía, UN La Pampa 22:13-21.

Colombo, A.; G.T. Pérez, P.D. Ribotta and A.E. León, 2008. A comparative study of physicochemical tests for quality prediction of Argentine wheat flours used as corrector flours and for cookie production. Journal of Cereal Sci. 48(3): 775-780.

Covas, G. 1976 Tricepiro, un nuevo verdeo sintético que involucra al trigo, centeno y agropiro. Informativo de Tecnol. Agrop. para la Región Semiárida Pampeana 68:5.

D’Appolonia, B. L. and P. Rayas-Duarte, 1994. Wheat carbohydrates: structure and functionality. In Bushuk, W. \& V. Rasper (Eds.). Wheat Production, Properties and Quality Capter 8 pp 107-127.

Duyvejonck, A.E.; B. Lagrain, E. Dornez, J.A. Delcour and C.M. Courtin, 2012. Suitability of solvent retention capacity test to assess the cookie and bread making quality of European wheat flours. LWT-Food Science and Technology 47(1):56-63.

Dick, J. and J. Quick, 1983. A modified screening test for rapid stimation of gluten strength in early-generation durum wheat breeding lines. Cereal Chemestry 60(4): 315-318.

Di Rienzo, J.; F. Casanoves, M. Balzarini, L. González, M. Tablada, y C. Robledo, 2015. InfoStat versión 2015. Grupo InfoStat, FCA, Universidad Nacional de Córdoba, Argentina. URL http://www.infostat.com.argentina.

Fairidi, H.; C. Gaines and P. Finney, 1994. Soft wheat quality in the production of cookies and crackers. In W. Bushuk and V. Rasper (Eds.) Wheat: Production, Properties and Quality. pp 154-168. Chapman and Hall, Glasgow, Scotland.

Ferreira, V.; E. Grassi, A. Ferreira, H. di Santo, E. Castillo, y H. Paccapelo, 2015. Triticales y tricepiros: interacción genotipo-ambiente y estabilidad del rendimento de grano. Chilean J. Agric. Anim. Sci. 31(2):93-104.

Gaines, C., 2000. Report of the AACC comittee on soft flour. Method 56-11, Solvent Retention Capacity Profile. Cereal Foods World 45:303-306.

Giroux, M.J. and C.F. Morris, 1997. A glycine change in puroindoline $b$ is associated with wheat grain hardness and low levels of starch-surface friabilin. Theor. Appl. Genet. 95(5-6):857-864.

Gómez, M.; L. Manchón, B. Oliete, E. Ruíz and P.A. Caballero, 2010. Adequacy of wholegrain non-wheat flours for layer cake elaboration. LWT- Food Science and Technology 43(3):507-513. 
Guerrero García, A., 1999. Cultivos herbáceos extensivos. Ed. Mundiprensa, Madrid. 833 pp.

Guttieri, M.J.; R. McLean, S.P. Lanning, L.E. Talbet and E.J. Souza, 2002. Assesing environmental influences on solvent retention capacities of two soft white spring wheat cultivars. Cereal Chemestry 79(6):880-884.

Guttieri, M.J. and E. Souza, 2003. Sources of variation in the solvent retention capacity test of wheat flour. Crop Sci. 43(5): 1628-1633.

Hashimoto, S.; M. Shrogren and Y. Pomeranz, 1987. Cereal pentosans: estimation and significance. I. Pentosans in wheat and milled wheat products. Cereal Chemistry 64(1): 30-34.

Hoseney, R.C., 1994. Principles of Cereal Science and Technology, $2^{\text {nd }}$ Ed., American Association of Cereal Chemists, Inc., St. Paul, Minnesota, USA.

Kweon, M.; L. Slade, H. Levine, R. Martin, L. Andrews and E. Souza, 2009. Effects of extent of chlorination, extraction rate and particle size reduction on flour and gluten functionality explored by solvent retention capacity (SRC) and mixograph. Cereal Chemistry 86(2):221-224.

León, A.E.; O.J. Rubiolo and M.C. Añon, 1996. Use of triticale in cookies: quality factors. Cereal Chemistry 73(6): 779- 784.

Miller, B.S.; S. Afework, Y. Pomeranz, B. Bruinsma and G.D. Booth, 1982. Measuring the hardness of wheat. Cereal Foods World 27:61-64.

Miller, B.S. and R.C. Hoseney, 1997. Factors in hard wheat flour responsible for reduced cookie spread. Cereal Chemestry 74(3):330-336.

Moiraghi, M.; P.D. Ribotta, A. Aguirre, G.T. Pérez y A.E. León, 2005. Análisis de la aptitude de trigos pan para la elaboración de galletitas y bizcochuelos. Agriscientia 22:47-54.

Oettler, G., 2005. The fortune of a botanical curiosity. Triticale: past, present and future. The Journal of Agricultural Science 143(5):329-346. Cambridge Univ. Press.

Oliete, B.; G.T. Pérez, M. Gómez, P.D. Ribotta, M. Moiraghi and A.E. León, 2010. Use of wheat, triticale and rye flours in layer cake production. International J. of Food Science \& Technology 45(4):697-796.

Olivera, A. and A. Baier, 1991. Evaluation of gluten quality and resistanse to scab, stop bloch and sproutin in triticale wheat and rye. In Proc. $2^{\text {nd }}$ International Triticale Sym. pp. 75-78. Berthier Grafica Editora. Passo Fundo, Brasil.

Pantanelli, A., 2002. Galletitas. Cadena alimentaria. Alimentos Argentinos 19:47-55.

Peña, R.J., 2003. Influencia de la textura del endospermo y la composición de las proteínas del gluten en la calidad panadera del trigo. En: Avances y perspectivas en calidad industrial del trigo. Acta INIA N ${ }^{\circ} 21$. Centro Regional de Investigación Carillanca. Temuco, Chile. pp 23-40.

Pérez, G.T.; A.E. León, P.D. Ribotta, A. Aguirre, O.J. Rubiolo y M.C. Añón, 2003. Use of triticale flours in cracker-making. European Food Res. Technol. 217(2):134137.

Ramírez, A.; G.T. Pérez, P.D. Ribotta y A.E. León, 2003. The occurrence of friabilins in triticale and their relationship with grain hardness and baking quality. J. Agric. Food Chem. 51(24):7161-7181.

Roccia, P.; M. Moiraghi, P.D. Ribotta, G.T. Pérez, O.J. Rubiolo and A.E. León, 2006. Use of solvent retention capacity profile to predict the quality of triticales flours. Cereal Chemistry 83(3):243-249

Rubiolo, O.J.; M. Moiraghi, P. Roccia Ruffinengo, G.T. Pérez y A.E. León, 2004. Evaluación de la calidad industrial de líneas avanzadas de triticale. VI Congreso Nacional de Trigo. Universidad Nacional del Sur. Actas CD.

Slade, L. and H. Levine, 1994. Structure-Function relationships of cookies and cracker ingredients. Pages 23-141. In Faridi, H. (Ed.). The Science of Cookie and Cracker Production. Chapman, New York.

Soil Survey Staff. 1999. Soil taxonomy: a basic system of soil classification for making and interpreting soil surveys. Agric. Handbook ( $2^{\text {nd }}$ ed.), USDA Vol. 436. 869 pp.

Torri, C.L., P.D. Ribotta, M.H. Morcillo, O.J. Rubiolo, G.T. Pérez y A.E. León, 2003. Determinación del contenido de almidón dañado en harinas de triticale. Su influencia sobre la calidad galletitera. Agriscientia 20: 3-8.

Vázquez, D., 2009. Aptitud industrial de trigo. INIA Serie Técnica No 177. R.O. del Uruguay. 46 pp.

Wade, P., 1988. Wheat and wheat flour. In Wade, P (Ed.): Biscuits, cookies and crackers. Vol. 1. The principles of the craft. pp 116-138. Elsevier Applied Science. London, England.

Williams, P.C., 1986. The influence of chromosome number and species on wheat hardness. Cereal Chemestry. 63(1):56-57.

Yamazaki, W.T. and D. Lord, 1971. Soft wheat products. In Pomeranz, Y. (Ed.): Wheat, chemistry and technology. $2^{\text {da }}$ Ed. 743-776. AACC, St. Paul. Minnesota, USA.

Yamazaki, W.T. and D. H. Donelson, 1972. Relationship between flour particle and cake volume potential among eastern soft wheat. Cereal Chemistry 49(6):649-653. 